\title{
CONTINUA WHICH HAVE WIDTH ZERO ${ }^{1}$
}

\author{
C. E. BURGESS
}

In a recent paper [7], the author showed that with each tree-like continuum there can be associated a non-negative number called the width of $M$, and it was shown that the plane, $E^{2}$, does not contain uncountably many disjoint tree-like continua each having a positive width. This result is used here in establishing some conditions under which a tree-like continuum in $E^{2}$ has width zero. There exists a treelike continuum, such as one which is the sum of a simple triod $T$ and a ray spiralling around $T$, that has width zero but one of its subcontinua has a positive width. Some of the theorems presented here give conditions under which a tree-like continuum $M$ has width zero hereditarily; that is, every subcontinuum of $M$ has width zero. ${ }^{2}$ While such a continuum has a "thinness" property similar to that of a chainable continuum, there do exist in $E^{2}$ tree-like continua, as indicated by Anderson [1], which have width zero hereditarily but are not chainable. The question in $\$ 4$ of [7] and Roberts' result [11] that every chainable continuum has uncountably many disjoint homeomorphic images in $E^{2}$ suggest the following questions. If the tree-like continuum $M$ is a subset of $E^{2}$ and has width zero hereditarily, does there exist a sequence of disjoint continua in $E^{2}$ converging homeomorphically to $M$ ? Does a tree-like continuum in $E^{2}$ have uncountably many disjoint homeomorphic images in $E^{2}$ if it has width zero hereditarily? ${ }^{3}$ These questions are not answered, but their converses are direct corollaries to some theorems in [7]. A tree-like continuum $M$ in $E^{2}$ has width zero hereditarily either if there exists a sequence of disjoint continua in $E^{2}$ converging homeomorphically to $M$ [7, Theorem 5] or if $M$ has uncountably many disjoint homeomorphic images in $E^{2}$ [7, Theorem 10].

In this paper, a compact connected metric space is called a continuum. Definitions of trees, chains, tree-like continua, and triods can be found in [6]. A definition of the width of a tree-like continuum is stated in [7], and the following property follows directly from this definition of width. A tree-like continuum $M$ has width zero if, and

\footnotetext{
Pr esented to the Society, September 1, 1961; received by the editors April 24, 1961

${ }^{1}$ Some of this work was supported by the National Science Foundation under G-5880.

2 This property was suggested by R. H. Bing during the discussion which followed the presentation of [7] at the Summer Meeting at East Lansing in August, 1960.

${ }^{3}$ It follows from Theorem 5 that this question is equivalent to one raised by Bing $[2$, p. 656]
} 
only if, for any cofinal sequence $T_{1}, T_{2}, \cdots$ of trees defining $M$, there exists, for each $i$, a chain $C_{i}$ in $T_{i}$ such that the sequence of sets $^{4} C_{1}^{*}, C_{2}^{*}, \cdots$ converges to $M$.

THEOREM 1. If $M$ is a tree-like continuum and for every positive number $\epsilon$ there is a subcontinuum of $M$ which has width zero and is $\epsilon-$ dense ${ }^{5}$ in $M$, then $M$ has width zero.

Proof. Let $K$ be a subcontinuum of $M$ which has width zero and is $\epsilon / 2$-dense in $M$. It follows from the above property of continua with width zero that there exists a positive number $\delta$ less than $\epsilon / 2$ such that every $\delta$-tree which is an essential covering of $K$ must contain a chain $C$ that is $\epsilon / 2$-dense ${ }^{6}$ in $K$. Now let $G$ be a $\delta$-tree that is an essential covering of $M$ and let $G^{\prime}$ be a subtree of $G$ that is an essential covering of $K$. Hence there is a chain $C^{\prime}$ in $G^{\prime}$ that is $\epsilon / 2$-dense in $K$, and it follows that $C^{\prime}$ is $\epsilon$-dense in $M$. This implies that $M$ has width zero.

THEOREM 2. If every proper subcontinuum of the tree-like continuum $M$ has width zero, then $M$ has width zero.

Proof. Let $\epsilon$ be a positive number, let $p_{1}, p_{2}, \cdots, p_{n}$ be distinct points of $M$ such that every point of $M$ is within a distance $\epsilon / 2$ of some $p_{i}$, and let $D_{1}, D_{2}, \cdots, D_{n}$ be open sets with disjoint closures such that, for each $i, D_{i}$ contains $p_{i}$ and has a diameter less than $\epsilon / 2$. Some subcontinuum $K$ of $M$ is irreducible with respect to the property of being a continuum which intersects the closures of all of the sets $D_{1}, D_{2}, \cdots, D_{n}$. It follows from [4, Theorem 3] that for some $i$, $K$ does not intersect $D_{i}$. Hence $K$ is a proper subcontinuum of $M$ and is $\epsilon$-dense in $M$. Since $K$ has width zero, it follows from Theorem 1 that $M$ has width zero.

COROLlaRY 2.1. If every proper subcontinuum of the tree-like continuum $M$ is chainable, then $M$ has width zero.

COROLlaRY 2.2. If every proper subcontinuum of the tree-like continuum $M$ is an arc, then $M$ has width zero.

THEOREм 3. In order that the nondegenerate tree-like continuum $M$ should have width zero, it is necessary and sufficient that $M$ be irreducible between some two points.

Proof of NeCessity. The continuum $M$ is not a triod [7, Theorem

\footnotetext{
4 The set which is the sum of the elements of $C_{i}$ is denoted by $C_{i}^{*}$.

5 A subset $H$ of $M$ is $\epsilon$-dense in $M$ if every point of $M$ is within a distance $\epsilon$ of $H$.

${ }^{6}$ This means that every point of $K$ is within a distance $\epsilon / 2$ of some link of $C$.
} 
3] and is unicoherent [3]. Sorgenfrey [12, Theorem 3.2] has shown that such a continuum is irreducible between some two points.

Proof of SUfficiency. Let $x$ and $y$ be two points such that $M$ is irreducible between them, and let $T_{1}, T_{2}, \cdots$ be a cofinal sequence of trees defining $M$. For each $i$, there is a chain $C_{i}$ in $T_{i}$ which covers both $x$ and $y$. Now some subsequence of $C_{1}^{*}, C_{2}^{*}, \ldots$ converges to a subcontinuum of $M$ that contains both $x$ and $y$. But no proper subcontinuum of $M$ contains both $x$ and $y$, so $C_{1}^{*}, C_{2}^{*}, \ldots$ converges to $M$. Hence $M$ has width zero.

Corollary 3.1. Every indecomposable tree-like continuum has width zero.

COROLlary 3.2. Every hereditarily indecomposable tree-like continuum has width zero hereditarily.

REMARK. Since a tree-like continuum has width zero hereditarily if it is either chainable or hereditarily indecomposable, one might wonder whether the converse is true. However, two continua of a type indicated by Anderson [1] can be joined at a point to obtain a tree-like continuum which is decomposable, has width zero hereditarily, and is not chainable. That every tree-like continuum with these three properties must contain a nondegenerate indecomposable continuum follows from the proof of the next theorem.

THEOREM 4. If every proper subcontinuum of the tree-like continuum $M$ is decomposable and has width zero, then every proper subcontinuum of $M$ is chainable.

Proof. Let $K$ be a proper subcontinuum of $M$. No triod has width zero [7, Theorem 3], so $K$ contains no triod. That $K$ is hereditarily unicoherent follows from the fact that this is a property of every treelike continuum [3]. Bing [2] has shown that a continuum is chainable if it is atriodic, hereditarily decomposable, and hereditarily unicoherent. Hence $K$ is chainable.

THEOREM 5. In order that a tree-like continuum $M$ should have width zero hereditarily, it is necessary and sufficient that $M$ should contain no triod.

Proof of NeCEssity. Every tree-like triod has a positive width [7, Theorem 3]. Hence no subcontinuum of $M$ is a triod.

Proof of sufficiency. Each subcontinuum of $M$ is unicoherent [3] and atriodic. Hence, as in the proof of Theorem 3, it follows from Sorgenfrey's theorem [12, Theorem 3.2] that each subcontinuum of 
$M$ is irreducible between some two points. Now by Theorem 3, each subcontinuum of $M$ has width zero.

THEOREM 6. Every homogeneous ${ }^{7}$ tree-like continuum in $E^{2}$ has width zero hereditarily.

Proof. Suppose that some homogeneous tree-like continuum $M$ in $E^{2}$ does not have width zero hereditarily. It follows from Theorem 2 that some proper subcontinuum $K$ of $M$ does not have width zero. F. B. Jones [9] has shown that every homogeneous tree-like continuum is indecomposable. Hence $M$ has uncountably many disjoint composants, ${ }^{8}$ and the homogeneity of $M$ implies that each of these composants contains a homeomorphic image of $K$. But no homeomorphic image of $K$ has width zero [7, Theorem 2], and this involves a contradiction since $E^{2}$ does not contain uncountably many disjoint tree-like continua with positive widths [7, Theorem 10]. Hence $M$ has width zero hereditarily.

TheоReм 7. If $M$ is a homogeneous continuum in $E^{2}$, then every proper subcontinuum of $M$ is tree-like and has width zero.

Proof. Every homogeneous continuum in $E^{2}$ is the boundary of each of its complementary domains [5, Theorem 2]. So each proper subcontinuum of $M$ fails to separate $E^{2}$ and hence is tree-like [2]. Now suppose that some proper subcontinuum $K$ of $M$ does not have width zero. The indecomposable case and the decomposable case will be considered separately, and a contradiction will be obtained in each case.

Case 1. If $M$ is indecomposable, then a contradiction can be obtained as in the proof of Theorem 6.

Case 2. If $M$ is decomposable, it follows from a theorem due to F. B. Jones [10] that there is a continuous collection $G$ of homogeneous indecomposable tree-like continua filling $M$ such that the decomposition space of $G$ is a simple closed curve. Hence it follows from Theorem 6 that each element of $G$ has width zero hereditarily, so that $K$ intersects at least two elements of $G$. Jones [10] has shown that each element of $G$ that intersects $K$ must be a subset of $K$. Hence there is a continuous subcollection $G^{\prime}$ of $G$ that fills $K$ so that the decomposition space of $G^{\prime}$ is an arc. Now it is a further consequence

7 A continuum $M$ is homogeneous if for each two points $x$ and $y$ of $M$ there is a homeomorphism of $M$ onto itself that carries $x$ into $y$.

${ }^{8}$ A subset $H$ of $M$ is said to be a composant of $M$ if, for some point $p$ of $M$, the set $H$ is the sum of all proper subcontinua of $M$ that contain $p$. Every nondegenerate indecomposable continuum has uncountable many disjoint composants [8]. 
of Jones' results in [10] that $K$ is irreducible from a point in one end element of $G^{\prime}$ to a point in the other end element of $G^{\prime}$. Hence it follows from Theorem 3 that $K$ has width zero.

\section{BiBLIOGRAPHY}

1. R. D. Anderson, Hereditarily indecomposable plane continua, Abstract 236, Bull. Amer. Math. Soc. 57 (1951), 185.

2. R. H. Bing, Snake-like continua, Duke Math. J. 18 (1951), 653-663.

3. R. H. Bing and F. B. Jones, Another homogeneous plane continuum, Trans. Amer. Math. Soc. 90 (1959), 171-192.

4. C. E. Burgess, Collections and sequences of continua in the plane, Pacific J. Math. 5 (1955), 325-333.

5. - Continua and various types of homogeneity, Trans. Amer. Math. Soc. 88 (1958), 366-374.

6. - Chainable continua and indecomposability, Pacific J. Math. 9 (1959), 653-659.

7. - Collections and sequences of continua in the plane. II, Pacific J. Math. 11 (1961), 447-454.

8. S. Janiszewski and C. Kuratowski, Sur les continus indécomposables, Fund. Math. 1 (1920), 210-222.

9. F. B. Jones, Certain homogeneous unicoherent indecomposable continua, Proc. Amer. Math. Soc. 2 (1951), 855-859.

10. - On a certain type of homogeneous plane continuum, Proc. Amer. Math. Soc. 6 (1955), 735-740.

11. J. H. Roberts, Concerning atriodic continua, Monatsh. Math. 37 (1930), 223230.

12. R. H. Sorgenfrey, Concerning triodic continua, Amer. J. Math. 66 (1944), 439460.

UNIVERSITY OF UTAH 